\title{
Stronger Security Model of Group Key Agreement
}

\author{
Jianjie Zhao ${ }^{1}$, Dawu Gu${ }^{2}$, and M. Choudary Gorantla ${ }^{3}$ \\ 1 School of Information Security Engineering, Shanghai Jiao Tong University. \\ Shanghai 200240, China. \\ 2 Department of Computer Science and Engineering, Shanghai Jiao Tong University. \\ Shanghai 200240, China. \\ 3 Society for Electronic Transactions and Security, CIT Campus, Taramani, Chennai \\ 600113, India. \\ jjzhao81@gmail.com, dwgu@sjtu.edu.cn, mc.gorantla@gmail.com
}

\begin{abstract}
In PKC 2009, Gorantla, Boyd and González Nieto presented a nice result on modelling security for group key agreement (GKA) protocols. They proposed a novel security model (GBG model) that better supports the adversaries' queries than previous models for GKA protocols by considering KCI resilience. However, ephemeral key leakage attack resistance has been left outside the scope of the GBG model. In this paper, we demonstrate an ephemeral key leakage on an existing GKA protocol which has been shown secure in the GBG model. We then extend the GBG model by allowing the adversary greater attack powers of leaking ephemeral keys in GKA protocol session. We also apply the well known NAXOS trick to propose an improvement to an existing GKA protocol, which can resist the ephemeral key leakage attack. The security of the improved protocol has been argued under the our new model.
\end{abstract}

Keywords: Group key agreement; Ephemeral key leakage attack; Security model; Provable security

\section{Introduction}

Recent rapid development in networking and related mass communication media and digital technology opens the extensive possibilities for group applications. Group applications represent a special case of multi-party applications where the participants have some unifying relationship concerning their rights, responsibility and application goals [1]. There are many well-known forms of group applications such as digital conferences, text-based group communication, file and data sharing etc. On the other hand, security is increasingly important for these applications where group members want to prevent outsiders from obtaining communication content which sometimes are confidential or sensitive. The detailed information on the security issue for group applications can be obtained in $[1]$. 
Group key establishment is an information security process that enables users establish a session key known only to them. This session key can be applied for subsequent cryptographic usage. Group key establishment can be divided in two different classes: group key distribution (GKD) and group key agreement (GKA). The main characteristic of a GKD protocol is that it needs some trusted party (either a third party or a group member) and some trusted transport channel. In contrast, GKA protocol allows all participants to establish the session key where no party is needed to choose as the key generator and distributor and no secure channel is required. In this paper, we restrict our research to GKA protocols.

Although there are many security models [2-8] for two or three-party key agreement protocols, the formal model proposed by Bresson, Chevassut, Pointcheval and Quisquater [9], which we refer to as the BCPQ model, is the first security model for GKA protocols. Following the work of Bresson, Chevassut and Pointcheval [10] (BCP model) that extended the BCPQ model to the dynamic membership case, they [11] revised the BCP model to meet the internal state information reveal resistance $(\mathrm{BCP}+)$ model. However, these models do not consider any malicious behaviour by the participants themselves. In CCS 2005, Katz and Shin [12] proposed a security model for GKA protocol, which provide the first formal treatment of security of GKA in the presence of malicious participants. Bohli, González-Vasco and Steinwandt [13] (BGS model) and Bresson and Manulis [14] (BM model) later proposed two extensions of the KS model under different corruption models. Additionally, the BGS and BM model deal with the issue of contributiveness in the presence of malicious insiders.

All the models above provide definitions that consider the requirements on indistinguishability of computed group keys and forward secrecy. However, as discussed by Gorantla et al. [15] the above security notions do not provide the key compromise impersonation (KCI) resilience. To demonstrate the importance of considering resistance to KCI attacks for GKA protocols, the authors presented KCI attacks on the protocols of Boyd and González Nieto [16], Al-Riyami and Paterson [17] and Bresson et al. [18]. They then proposed a new model (GBG model) by taking KCI resilience into account and also showed that the protocol of Bohli et al. [13] (BGS protocol) to be secure in the GBG model.

Inspired by Manulis et al.'s recent work [19] where the authors pointed out that the GKA security models have so far not considered leakage of ephemeral keys, we augment the GBG model by considering ephemeral key leakage. In our new model, we allow the adversary to reveal any long-term key and ephemeral key of participants involved except for both long-term key and ephemeral key of one of the participants of the test session. We then improve the BGS protocol and show that it is secure in the new model. More specially, we have the following results:

1. A stronger security model for GKA protocols. By updating the definitions of adversary queries and notion of freshness of the GBG model, we propose a new model for GKA protocols which provides the adversary more attack capabilities than those in the earlier models. 
2. An attack on the BGS protocol and its improvement. We first show that the BGS protocol is not secure in our new security model. To avoid this drawback, a solution where the ephemeral secret result will be generated by the hash function of the long-term key and the ephemeral key is put forward. This technique is known as the NAXOS trick in the literature [4].

3. A straightforward security proof in the new model. Under some well-studied assumptions, we prove the improved BGS protocol is secure in our new model.

We begin with a brief review of the GBG model in Section 2.1 and discuss the attack not covered by their definition in Section 2.2, then we give a simple ephemeral key leakage attack on the BGS protocol using this security flaw in the remainder of this section. Section 3 introduces our extended GBG model. In Section 4, we present an improved version of the BGS protocol which can resist the attack discussed in Section 2.2. The formal security argument is given in Section 5 .

\section{The GBG model and the BGS protocol}

In this section, we overview the GBG model briefly and point out that the ephemeral key leakage attack is not covered by it. Then we present this attack on the BGS protocol which is provably secure in the GBG model.

\subsection{Overview of GBG model}

The GBG model [15] provides a formal security assurance to GKA protocols. We give a high-level overview of the GBG model in this subsection.

Participants. A GKA protocol runs in a network of multiple interconnected participants where each participant is activated to run sessions for itself and its peers. As a result, participants in such a session establish a key called a session key. We denote the participant set by $\mathcal{U}=\left\{U_{1}, U_{2}, \cdots, U_{n}\right\}$ and the protocol may be run among any subset of these parties. In a GKA protocol, each participant may execute a polynomial number of protocol instances in parallel. We refer to the $i$-th instance of $U \in \mathcal{U}$ as $\prod_{U}^{i}$.

Adversary model. The communication network is assumed to be fully controlled by an adversary $M$, that is, it may eavesdrop, delay, alter and insert messages during the process of the protocol at will. We define the security of a GKA protocol by a series of games between a challenger and the adversary $M$ (an outsider adversary or a malicious insider) in which the adversary must solve a challenge on a test session. In this games, $M$ is allowed to select the identities of all the honest participants and ask the following queries in any sequence.

- Execute $\left(\prod_{U}^{i}\right)$. The adversary makes this query to obtain the protocol messages that were exchanged during the honest execution $\left(\prod_{U}^{i}\right)$ of the protocol. This query models the passive attacks. 
- Send $\left(\prod_{U}^{i}, m\right)$. The adversary makes this query to obtain the message that the instance $\prod_{U}^{i}$ would generate on receipt of the message $m$.

- RevealKey $\left(\prod_{U}^{i}\right)$. The adversary makes this query to obtain the session key established at the accepted instance $\prod_{U}^{i}$.

- $\operatorname{Corrup}(U)$. The adversary makes this query to obtain the long-term key of the participant $U$.

- RevealState $\left(\prod_{U}^{i}\right)$. The adversary makes this query to obtain the internal state of $\left(\prod_{U}^{i}\right)$. We assume that the internal state is erased once $\prod_{U}^{i}$ has accepted. Hence, a RevealState query to an accepted instance returns nothing.

- Test $\left(\prod_{U}^{i}\right)$. Only one query of this type is allowed for the adversary. Provided that $\prod_{U}^{i}$ is accepted, the adversary $M$ can make this query at any time. To respond to this query, a random bit $b \in\{0,1\}$ is secretly selected. If $b=1$, then the real session key established at $\left(\prod_{U}^{i}\right)$ is returned. Otherwise, a uniformly chosen random value from the session key distribution is returned.

We now define the AKE security, the MA security and the Contributiveness based on the definitions of partner and freshness.

Definition 1 (Partner). Informally, an instance $\prod_{U}^{i}$ is said to be accepted if it goes into an accept state after receiving the last expected protocol message, that is, when an instance accepts, it holds a session key, a session identifier sid and a partner identifier pid. The information of whether an instance has terminated with acceptance or not is assumed to be known to the adversary. Two instances $\prod_{U}^{i}$ and $\prod_{U^{\prime}}^{j}$ at two different parties $U$ and $U^{\prime}$ respectively are considered partnered iff

1. Both the instances have accepted.

2. The session identifiers of $\prod_{U}^{i}$ and $\prod_{U^{\prime}}^{j}$ are the same.

3. The partner identifiers of $\prod_{U}^{i}$ and $\prod_{U^{\prime}}^{j}$ are the same.

Definition 2 (Freshness). An instance $\prod_{U}^{i}$ is fresh if the following conditions hold:

1. The instance $\prod_{U}^{i}$ or any its partner has not been asked a RevealKey query after their acceptance.

2. The instance $\prod_{U}^{i}$ or any its partner has not been asked a RevealState query before their acceptance.

3. If $\prod_{U^{\prime}}^{j}$ is a partner of $\prod_{U}^{i}$ and $M$ asked Corrupt $\left(U^{\prime}\right)$, then any message that $M$ sends to $\prod_{U}^{i}$ on behalf of $\prod_{U^{\prime}}^{j}$ must come from $\prod_{U^{\prime}}^{j}$ intended to $\prod_{U}^{i}$.

Definition 3 (AKE security). In Stage 1, an adversary $M_{A K E}$ against the AKE security notion is allowed to make all the queries defined above. $M_{A K E}$ makes Test query to an instance $\prod_{U}^{i}$ at the end of Stage 1 and is given a challenge key $K_{b}$. It can continue asking queries in Stage 2. Finally, $M_{A K E}$ outputs a bit 
$b^{\prime}$ and wins the AKE security game if (1) $b=b^{\prime} ;(2)$ the instance $\prod_{U}^{i}$ that was asked Test query remained fresh till the end of $M_{A K E}$ 's execution. We define the advantage of $M_{A K E}$ in winning the AKE security game as $A d v_{M_{A K E}}=$ $\left|2 \cdot \operatorname{Pr}\left[b^{\prime}=b\right]-1\right|$. A protocol is called AKE secure if $A d v_{M_{A K E}}$ is negligible in the security parameter $k$.

Definition 4 (MA security). An adversary $M_{M A}$ against the MA security notion is allowed to make all the queries defined above. $M_{M A}$ violates the mutual authentication property of the GKA protocol if at some point during the protocol run, there exists an uncorrupted instance $\prod_{U}^{i}$ that has accepted with a session key $s k_{U}^{i}$ and another party $U^{\prime} \in p i d_{U}^{i}$ that is uncorrupted at the time $\prod_{U}^{i}$ accepts such that

1. There is no instance $\prod_{U^{\prime}}^{j}$ with $\left(p i d_{U^{\prime}}^{j}, \operatorname{sid}_{U^{\prime}}^{j}\right)=\left(p i d_{U}^{i}, \operatorname{sid}_{U}^{i}\right)$ or

2. There is an instance $\prod_{U^{\prime}}^{j}$ with $\left(p_{i d_{U^{\prime}}^{j}}^{j}, \operatorname{sid}_{U^{\prime}}^{j}\right)=\left(p i d_{U}^{i}\right.$, sid $\left.{ }_{U}^{i}\right)$ that has accepted with $s k_{U^{\prime}}^{j} \neq s k_{U}^{i}$.

We define the probability of $M_{M A}$ in winning the MA security game as $A d v_{M_{M A}}$. A protocol is said to provide mutual authentication in the presence of insiders if $A d v_{M_{M A}}$ is negligible in $k$.

Definition 5 (Contributiveness [14]). An adversary $M_{C o n}$ against the contributiveness notion is allowed to make all the queries defined above. It operates in two stages prepare and attack as follows:

prepare. $M_{C o n}$ queries the instance $\prod$ and outputs some state information $\zeta$ along with a key $\tilde{k}$.

At the end of prepare stage, a set $\sum$ is built such that $\sum$ consist of uncorrupted instances which have been asked either Execute or Send queries.

attack. On input $\left(\zeta, \sum\right), M_{C o n}$ interacts with the instances of $\prod$ as in the prepare stage.

At the end of this stage $M_{C o n}$ outputs $(U, i)$ and wins the games if an instance $\prod_{U}^{i}$ at an uncorrupted party $U$ has terminated accepting $\tilde{k}$ with $\prod_{U}^{i} \notin \sum$.

We define the probability of $M_{C o n}$ in winning the above game as $A d v_{M_{C o n}}$. A protocol is said to provide contributiveness property in the presence of insiders if $A d v_{M_{C o n}}$ is negligible in $k$.

\subsection{An attack not covered by the GBG model}

We focus on Condition 2 in the definition of "Freshness" (Definition 2) and point out an attack which may compromise the security of GKA protocols proven secure in the GBG model. According to this condition, the GBG model does not allow the adversary to make RevealState query against the test session which it wants to attack. In other words, the GBG model does not provide any security guarantees for a session if the ephemeral key of either party has been leaked. To avoid such an attack (we call this attack the ephemeral key leakage (EKL) attack), GBG model assumes that the internal state should be erased after $\prod_{U}^{i}$ has accepted. However, this restriction prevents the adversary from revealing 
the ephemeral keys of participants during the protocol execution process, that is, the GBG model restricts the adversary's ability to attack the objective GKA protocol using the revealed ephemeral key during the protocol execution process. Next, we will present a concrete EKL attack on the BGS protocol which has been shown secure in the GBG model.

\subsection{EKL attack on BGS protocol}

The BGS protocol [13] proposed by Bohli et al. has been shown to satisfy their definitions of outsider and insider security. We briefly review the protocol here.

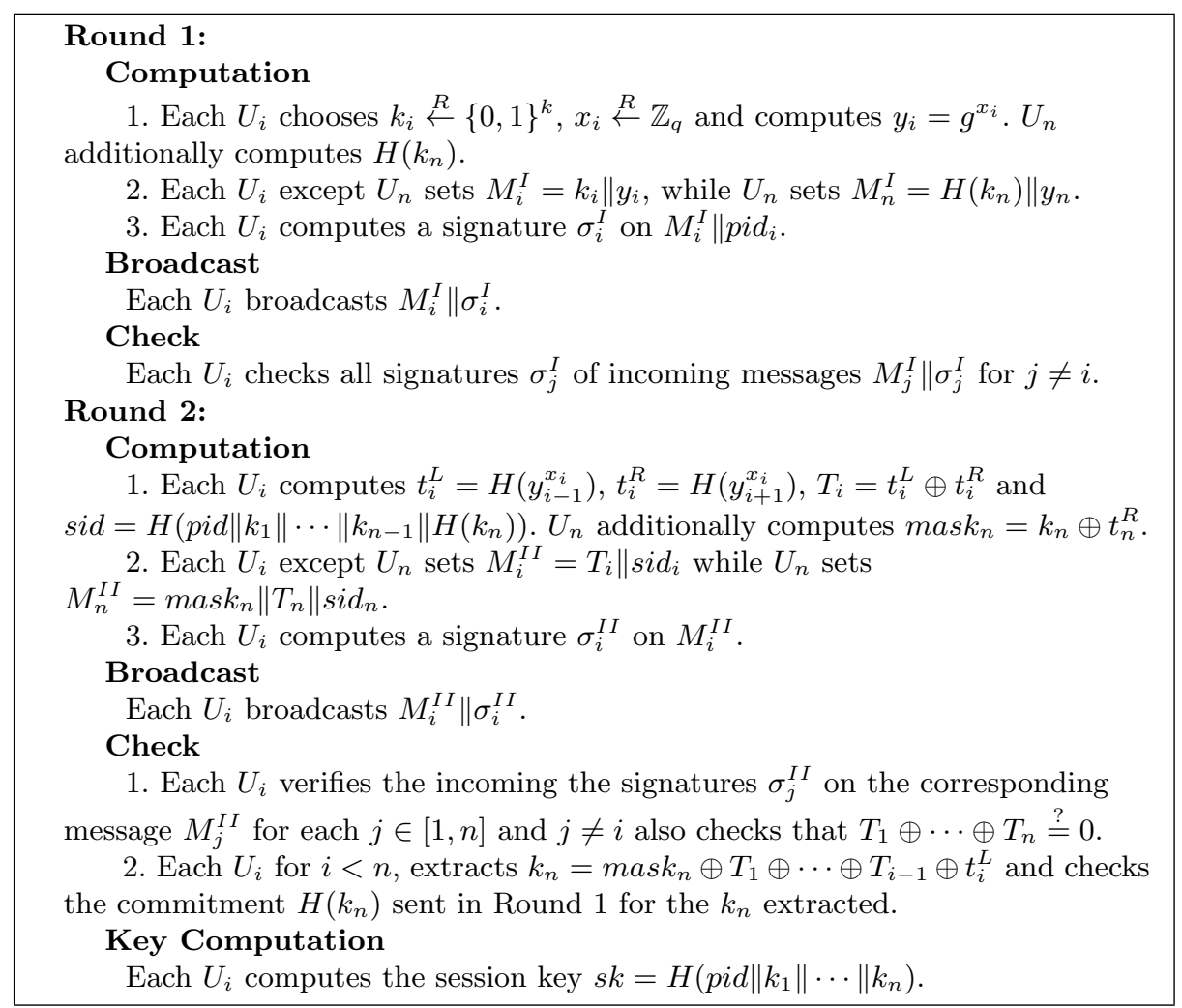

Fig. 1. BGS protocol

Let $\mathcal{U}=\left\{U_{1}, U_{2}, \cdots, U_{n}\right\}$ be the set of the parties who want to establish the session key. Suppose that the group members are ordered in a logical ring with $U_{i-1}$ and $U_{i+1}$ being the left and right neighbors of $U_{i}$ for $1 \leq i \leq n, U_{0}=U_{n}$ and $U_{n+1}=U_{1}$. During the initialization phase, a cyclic group $\mathbb{G}$ of prime order $q$, an arbitrary generator $g$ of $\mathbb{G}$ and the description of a hash function $H$ that 
maps to $\{0,1\}^{k}$ are chosen. Each party is assumed to have a long-term key pair for public signature. Figure 1 shows the execution process after initialization phase.

We show that BGS protocol is vulnerable to the EKL attack. Without loss of generality, we assume that the ephemeral key of $U_{i}$ is leaked, an adversary $M$ can compute $t_{i}^{L}=H\left(y_{i-1}^{x_{i}}\right)$ and $t_{i}^{R}=H\left(y_{i+1}^{x_{i}}\right)$ in Round 2. Using the public mask $_{n}, T_{1}, T_{2}, \cdots, T_{i-1}$ and its own $t_{i}^{L}, M$ will extract $k_{n}=$ mask $_{n} \oplus T_{1} \oplus T_{2} \oplus$ $\cdots \oplus T_{i-1} \oplus t_{i}^{L}$ and $s k=H\left(p i d\left\|k_{1}\right\| \cdots \| k_{n}\right)$. In this way, $M$ easily win the AKE game by selecting the test session at $U_{i}$.

A straightforward solution is to delete $x_{i}$ of participant $U_{i}$ during the protocol execution process, but it is infeasible. In more specific terms, it is impossible for $U_{i}$ to delete $x_{i}$ after Round 1 , since $U_{i}$ has to use $x_{i}$ in Round 2 to compute $t_{i}^{L}$ and $t_{i}^{R}$; if $U_{i}$ deletes $x_{i}$ after Round 2, the unavoidable leakage of $x_{i}$ during the first round could destroy the security of BGS protocol.

Note: It is worthy of being mentioned that BGS protocol is secure in GBG model since according to the principle of GBG model, all the ephemeral $x_{i}$ s will be erased after the corresponding session completed. Here we would like to reiterate that the attack not covered in the GBG model.

\section{Extended GBG model}

We now present our extended GBG (eGBG) model.

Participants. A GKA protocol runs in a network of multiple interconnected participants where each participant is activated to run sessions for itself and its peers. As a result, participants in such a session establish a session key. We denote the participant set by $\mathcal{U}=\left\{U_{1}, U_{2}, \cdots, U_{n}\right\}$ and the protocol may be run among any subset of these parties. In a GKA protocol, each participant may execute a polynomial number of protocol instances in parallel. We refer to the $i$-th protocol instance at $U \in \mathcal{U}$ as $\prod_{U}^{i}$.

Adversary model. The communication network is assumed to be fully controlled by an adversary $M$, that is, it may eavesdrop, delay, alter and insert messages during the process of the protocol at will. We define the security of a GKA protocol by a series of games between a challenger and the adversary $M$ (an outsider adversary or a malicious insider) in which the adversary must solve a challenge on a test session. In this games, $M$ is allowed to select the identities of all honest participants and issue the following queries in any sequence.

- Execute $\left(\prod_{U}^{i}\right)$. The adversary makes this query to obtain the message that were exchanged during the honest execution $\left(\prod_{U}^{i}\right)$ of the protocol. This query models the passive attacks.

- Send $\left(\prod_{U}^{i}, m\right)$. The adversary makes this query to obtain the message that the instance $\prod_{U}^{i}$ would generate on receipt of the message $m$. 
- RevealKey $\left(\prod_{U}^{i}\right)$. The adversary makes this query to obtain the session key established at the accepted $\prod_{U}^{i}$.

- Long-termKeyReveal $(U)$. The adversary makes this query to obtain the long-term key of the participant $U$.

- EphemeralKeyReveal $\left(\prod_{U}^{2}\right)$. The adversary makes this query to obtain the ephemeral key of $U$ in the $i$-th run.

- $\operatorname{Test}\left(\prod_{U}^{i}\right)$. Only one query of this form is allowed for the adversary. Provided that $\prod_{U}^{i}$ is accepted, the adversary $M$ can make this query at any time. To respond to this query, a random bit $b \in\{0,1\}$ is selected. If $b=1$, then the real session key is returned. Otherwise, a random value uniformly chosen from the session key distribution is returned.

We now define the notion of freshness according to the same definition of partnership in the GBG model.

Definition 6 (Freshness). An instance $\prod_{U}^{i}$ is fresh if the following conditions hold:

1. The instance $\prod_{U}^{i}$ or any its partner has not been asked a RevealKey query after their acceptance.

2. The instance $\prod_{U}^{i}$ or any its partner has not been asked a Long-termKeyR eveal query and a EphemeralKeyReveal query simultaneously before their acceptance. That is, the adversary is allowed to reveal any subset of four pieces of secret information (the long-term keys and the ephemeral keys of the participant and its partner) which does not contain both the long-term and ephemeral keys of one party.

3. If $\prod_{U^{\prime}}^{j}$ is a partner of $\prod_{U}^{i}$ and $M$ asked Long-termKeyReveal $\left(U^{\prime}\right)$, then any message that $M$ sends to $\prod_{U}^{i}$ on behalf of $\prod_{U^{\prime}}^{j}$ must come from $\prod_{U^{\prime}}^{j}$ intended to $\prod_{U}^{i}$.

The description of the AKE security, MA security and Contributiveness are the same as that in the GBG model in Section 2.1.

Compared to the GBG model, we

1. Replace the RevealState query with the EphemeralKeyReveal by which the adversary can get the ephemeral key of the instance $\prod_{U}^{i}$. At the same time, we remove the limitation of the original GBG model where once $\left(\prod_{U}^{i}\right)$ has accepted the internal state is erased.

2. Replace the Corrupt query with the Long-termKeyReveal to give the adversary the power to reveal the long-term keys.

3. Modify the Condition 2 in the notion of "freshness" to expand the attack scope of the adversary.

Remark 1. In many security models, when the adversary corrupts an honest party, it takes full control over this party and reveals all its secret information. In the GBG model, since the Corrupt query just simulates the long-term key leakage attack, we change the tag of this query to capture the adversarial ability more accurately. The role of Long-termKeyReveal in our model is the same as that of Corrupt in the GBG model. 
Overall, eGBG model extends the adversarial capabilities to the following extent: we allow the adversary to reveal any subset of the long-term keys and ephemeral keys of $\prod_{U}^{i}$ and its partner which does not contain both the long-term and ephemeral keys of one party.

\section{BGS+ protocol}

To construct a GKA protocol which is secure in eGBG model, we modify some details of the original BGS protocol and get BGS+ protocol.

\subsection{Description of BGS+ protocol}

We first introduce some notation that will be used in the rest of the paper.

$\mathcal{U}=\left\{U_{1}, U_{2}, \cdots, U_{n}\right\}$ : The set of the parties who want to establish the session key. We also suppose that the group members are ordered in a logical ring.

$p, q, g$ : Two large primes $p$ and $q$ with $q \mid(p-1)$, and a generator $g$ of group $\mathbb{G}$ with order $q$.

$k$ : The security parameter in this protocol.

$\left(p k_{i}, s k_{i}\right)$ : The long-term key pair of the participant $U_{i}$ which is used for the signature.

$k_{i}, x_{i}$ : The ephemeral keys pair of the participant $U_{i}$.

$H:\{0,1\}^{*} \rightarrow\{0,1\}^{\lambda}$ : Hash function modelled as a random oracle, where $\lambda$ is another security parameter.

$H_{1} / H_{2}:\{0,1\}^{*} \rightarrow Z_{q} / Z_{p}$ : Hash functions modelled as random oracles.

$S K$ : Session key established by the participants.

This protocol aims to establish a confidential communication. The detailed description can be seen in Fig.2. Note that we drop the operator "modp" for clarity.

\subsection{Some design principles}

The most significant difference between BGS protocol and ours is that we use $\widetilde{x_{i}}=H_{1}\left(s k_{i}, x_{i}\right)$ instead of $x_{i}$ as the ephemeral intermediate result to generate $y_{i}, t_{i}^{L}$ and $t_{i}^{R}$. This benefit of doing so is obvious: since we will destroy $\widetilde{x_{i}}$ after getting the needed values, this method avoid the possibility that the ephemeral secret state will be obtained by the adversary during the protocol execution process. On the other hand, even if the adversary gets the ephemeral key $x_{i}$, it can not obtain the $\widetilde{x_{i}}$ yet since it has to get the long-term key $s k_{i}$ at the same time, but it is not allowed to reveal the long-term key and the ephemeral key simultaneously in eGBG model.

\section{Security Analysis}

Now, we prove that BGS+ protocol achieves the AKE security goal, the MA security goal and the Contributiveness under reasonable and well-defined intractability assumptions. 


\section{Round 1:}

\section{Computation}

1. Each $U_{i}$ chooses $x_{i} \stackrel{R}{\leftarrow} \mathbb{Z}_{q}$ and computes $\widetilde{x_{i}}=H_{1}\left(s k_{i}, x_{i}\right)$ and $y_{i}=g^{\widetilde{x_{i}}}$. After obtaining $y_{i}, U_{i}$ destroys $\widetilde{x_{i}}$.

2. Each $U_{i}$ sets $M_{i}^{I}=y_{i} \| p i d$.

3. Each $U_{i}$ computes a signature $\sigma_{i}^{I}$ on $M_{i}^{I}$.

Broadcast

Each $U_{i}$ broadcasts $M_{i}^{I} \| \sigma_{i}^{I}$.

\section{Round 2:}

\section{Check}

Each $U_{i}$ checks all signatures $\sigma_{j}^{I}$ of incoming messages $M_{j}^{I} \| \sigma_{j}^{I}$ for $j \neq i$.

\section{Computation}

1. Each $U_{i}$ computes $\widetilde{x_{i}}=H_{1}\left(s k_{i}, x_{i}\right), t_{i}^{L}=H_{2}\left(\widetilde{x_{i-1}}\right), t_{i}^{R}=H_{2}\left(\widetilde{x_{i+1}}\right)$ and $T_{i}=t_{i}^{L} \oplus t_{i}^{R}$.

2.Each $U_{i}$ chooses $k_{i} \stackrel{R}{\leftarrow}\{0,1\}^{k}$. Then $U_{i}$ except $U_{n}$ computes $M_{i}^{I I}=T_{i} \| k_{i}$. $U_{n}$ computes mask $_{n}=k_{n} \oplus t_{n}^{R}$ and $H_{2}\left(k_{n}\right)$ and sets $M_{n}^{I I}=$ mask $_{n}\left\|T_{n}\right\| H_{2}\left(k_{n}\right)$. After this step, each $U_{i}$ destroys $\widetilde{x_{i}}, t_{i}^{L}$ and $t_{i}^{R}, U_{n}$ additionally destroys $k_{n}$.

3. Each $U_{i}$ computes a signature $\sigma_{i}^{I I}$ on $M_{i}^{I I}$.

\section{Broadcast}

Each $U_{i}$ broadcasts $M_{i}^{I I} \| \sigma_{i}^{I I}$.

After Round 1 and Round 2

\section{Check}

1. Each $U_{i}$ verifies the incoming the signatures $\sigma_{j}^{I I}$ on the corresponding message $M_{j}^{I I}$ for each $j \in[1, n]$ and $j \neq i$ also checks that $T_{1} \oplus \cdots \oplus T_{n} \stackrel{?}{=} 0$.

2. Each $U_{i}$ for $i<n$, computes $\widetilde{x_{i}}=H_{1}\left(s k_{i}, x_{i}\right)$ and $t_{i}^{L}=H_{2}\left(\widetilde{x_{i-1}}\right)$, extracts $k_{n}=\operatorname{mask}_{n} \oplus T_{1} \oplus \cdots \oplus T_{i-1} \oplus t_{i}^{L}$ and checks the commitment $H_{2}\left(k_{n}\right)$ sent in Round 2 for the $k_{n}$ extracted. If so, each $U_{i}$ computes sid $=H_{2}\left(\right.$ pid $\left.\left\|k_{1}\right\| \cdots\left\|k_{n-1}\right\| H_{2}\left(k_{n}\right)\right)$.

\section{Key Computation}

Each $U_{i}$ computes the session key $S K=H\left(\operatorname{sid}\left\|k_{1}\right\| \cdots \| k_{n}\right)$.

Fig. 2. BGS+ protocol 
Theorem 1 (AKE security). Let $H_{1}, H_{2}$ and $H$ be three random oracles and the signature scheme used in GBS+ is UF-CMA secure, $M_{A K E}$ denotes an adversary that has the ability to perform the oracle queries mentioned in Section 3. Then BGS+ is AKE secure if the CDH assumption holds in $G$. Furthermore, suppose $M_{A K E}$ has an advantage $A d v_{B G S+}^{A K E}\left(M_{A K E}\right)$ to break AKE security of BGS+ by activating at most $l$ sessions for each party, then there is a CDH problem solver $W$ that can solve the $\mathrm{CDH}$ problem in polynomial time with an advantage $A d v_{B G S+}^{C D H}(W)$ and

$$
A d v_{B G S+}^{A K E}\left(M_{A K E}\right) \leq \frac{q_{s}^{2}+q_{H_{2}}}{2^{k-1}}+\frac{q_{H_{1}}^{2}}{q}+\frac{q_{H_{2}}^{2}}{p}+\frac{q_{H}^{2}}{2^{\lambda}}+2 n^{2} \cdot A d v_{B G S+}^{S i g}+2 n l .
$$
$A d v_{B G S+}^{C D H}(W)$, where $n$ is the number of parties; $A d v_{B G S+}^{S i g}$ denotes the advantage of a polynomial adversary against the UF-CMA security of the signature; $q_{H_{1}}$, $q_{H_{2}}$ and $q_{H}$ are the maximum number of times of hash queries $H_{1}, H_{2}$ and $H$, respectively; $q_{s}$ represents the maximum number of queries to the Send oracles asked by the adversary.

Proof. In this proof, we incrementally define a sequence of games starting at the real execution $G 0$ and ending up at game $G 5$ where the adversary has no advantage. Each game addresses a different security aspect. For each game $G_{i}$, we define $\operatorname{Succ}_{i}(i=0,1, \cdots, 5)$ as the event that $M_{A K E}$ wins the game.

We use the game hopping technique based on the failure events and bridging steps suggested by Shoup [20]. Consider an event E that may occur during the adversary $M_{A K E}$ 's execution such that $\mathrm{E}$ is detectable by the simulator, $\mathrm{E}$ is independent of $\operatorname{Succ}_{i}, G_{i}$ and $G_{i+1}$ are identical unless E occurs, then we have

$$
\mid \operatorname{Pr}\left[\text { Succ }_{i+1}\right]-\operatorname{Pr}\left[\text { Succ }_{i}\right] \mid \leq \operatorname{Pr}[\mathrm{E}] .
$$

Game G0. This game corresponds to the real execution. In the game, we don't modify the simulation of the oracles, all the instances modeled as the real execution in the random oracle model. By the definition, we have

$$
A d v_{B G S+}^{A K E}\left(M_{A K E}\right)=\left|2 \cdot \operatorname{Pr}\left[S u c c_{0}\right]-1\right| .
$$

Game $G 1$. This game is as the same as the game $G 0$ except that the simulation fails if the event Forge happens.

The event Forge occurs when the adversary $M_{A K E}$ successfully forgery of one of the honest parties' signatures. According to the analysis in Gorantla et al.'s work [15], if the case happens, it will contradict against the assumption that the signature scheme in BGS+ protocol is UF-CMA secure.

We denote $A d v_{B G S+}^{S i g}$ be the advantage of a polynomial adversary against the UF-CMA security of the signature, since the probability that $M_{A K E}$ doesn't get the long-term key of the target party is $\geq \frac{1}{n}$ and the probability of $M_{A K E}$ outputting a valid forgery on behalf of the target party is also $\geq \frac{1}{n}$, we have $A d v_{B G S+}^{S i g} \geq \frac{1}{n^{2}}$, then

$$
\operatorname{Pr}\left[\text { Succ }_{1}\right]-\operatorname{Pr}\left[\text { Succ }_{0}\right] \leq \operatorname{Pr}[\text { Forge }] \leq n^{2} \cdot A d v_{B G S+}^{S i g} .
$$

Game $G 2$. In this game, we simulate all oracles in $G 1$, except that we halt all executions in which the event Collision occurs. 
The event Collision occurs when the random oracles $H_{1}, H_{2}$ and $H$ produce collisions for any of their own inputs. According to the birthday paradox, the probability of collisions in output of $H_{1}$ and $H_{2}$ oracles are at most $q_{H_{1}}^{2} /(2 q)$ and $q_{H_{2}}^{2} /(2 p)$ respectively where $q_{H_{i}}$ for $i=1,2$ denotes the maximum number of queries to $H_{i}$. Similarly, the probability of collision in output of $H$ oracle is at most $q_{H}^{2} / 2^{\lambda+1}$, where $q_{H}$ is the maximum number of times of hash queries $H$. Consequently,

$$
\mid \operatorname{Pr}\left[\text { Succ }_{2}\right]-\operatorname{Pr}\left[\text { Succ }_{1}\right] \mid \leq \operatorname{Pr}[\text { Collision }] \leq \frac{q_{H_{1}}^{2}}{2 q}+\frac{q_{H_{2}}^{2}}{2 p}+\frac{q_{H}^{2}}{2^{\lambda+1}} .
$$

Game G3. This game is as the same as the previous game except that the simulation fails if the event Repeat occurs.

The event Repeat occurs when different parties choose the same random value $k_{i}$ as their own shares. Let $q_{s}$ be the maximum number of queries to the Send oracles asked by the adversary, then $\operatorname{Pr}$ [Repeat] $\leq \frac{q_{s}^{2}}{2^{k}}$. Therefore, we have

$$
\mid \operatorname{Pr}\left[\operatorname{Succ}_{3}\right]-\operatorname{Pr}\left[\text { Succ }_{2}\right] \mid \leq \operatorname{Pr}[\text { Repeat }] \leq \frac{q_{s}^{2}}{2^{k}}
$$

Game G4. In this game, we change the simulation of queries to the Send query. This time, we change the way we compute the value $y_{i-1}$ in some manner (we will describe these ways subsequently) to replace $y_{i-1}$ when the adversary makes the corresponding Send query. Then we will have

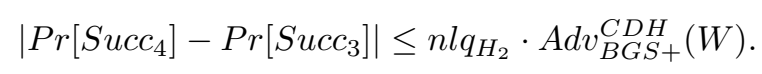

Proof. By assuming a successful adversary against this game, we can construct a CDH solver $W$.

There is only difference between $G 3$ and $G 4$ in the way to generate $y_{i-1}$ and $y_{i}$ for a session, we will show the difference between the current game and the previous one is negligible as long as the CDH assumption holds.

First, the CDH solver $W$ obtains CDH tuple $\left(g, g^{a}, g^{b}\right)$. As the real execution, $W$ randomly select a party $U_{i}(i=1,2, \cdots, n)$ and a session executed by it as the test session. When the corresponding Send query is asked, the CDH solver sets $A=y_{i-1}$ and $B=y_{i}$ and returns $A$ and $B$ to $U_{i}$. For other queries, $W$ returns as $G 3$.

Let $\operatorname{Pr}_{1}\left(M_{A K E}\right)$ be the probability of the event that $M_{A K E}$ will select $U_{i}$ and the corresponding chosen by $W$ as the test session, since there are $n$ parties and each party can active at most $l$ sessions, we have $\operatorname{Pr}_{1}\left(M_{A K E}\right) \geq \frac{1}{l n}$.

By the definition of freshness, the adversary is allowed to reveal any subset of long-term keys and ephemeral keys of the parties, but it is not allowed to get both the long-term keys and ephemeral keys of one party. Special for the party $U_{i}$, eGBG model allows the adversary to reveal $s k_{i}$ or $x_{i}$, but doesn't allow to reveal both $s k_{i}$ and $x_{i}$. Therefore, the $\mathrm{CDH}$ solver simulates all oracle queries without knowing $a$ and $b\left(a=\widetilde{x_{i}}\right.$ and $\left.b=\widetilde{x_{i-1}}\right)$.

Following the simulation principle above, if $M_{A K E}$ successfully violates the AKE security of BGS+ protocol, it must have obtained $k_{n}=\operatorname{mask}_{n} \oplus T_{1} \oplus \cdots \oplus$ 
$T_{i-1} \oplus t_{i}^{L}$ and know the value $t_{i}^{L}=H_{2}\left(y_{i-1}^{\widetilde{x_{i}}}\right)=H_{2}\left(\widetilde{y_{i}} \widetilde{x_{i-1}}\right)$. Since we assume $H_{2}$ is modeled as a random oracle, to get $t_{i}^{L}$, the only way for $M_{A K E}$ is to own $y_{i-1}^{\widetilde{x_{i}}}$ $\left(y_{i}^{\widetilde{x_{-1}}}\right)$ and ask Hash query $\left(H_{2}\right)$. Once it asks Hash query $\left(H_{2}\right)$, it has to leave the correct $\widetilde{y_{i-1}}\left(y_{i}^{\widehat{x_{i-1}}}\right)$.

With the probability $\operatorname{Pr}_{2}\left(M_{A K E}\right) \geq \frac{1}{q_{H_{2}}}, W$ can choose the appropriate $y_{i-1}^{\widetilde{x_{i}}}\left(\widetilde{x_{i}}\right)$ and solve the $\mathrm{CDH}$ problem with the advantage $A d v_{B G S+}^{C D H}(W)$ in polynomial time: $C D H(A, B)=C D H\left(g^{a}, g^{b}\right)=y_{i-1}^{\widetilde{x_{i}}}=y_{i}^{\widetilde{x_{i-1}}}=g^{a b}$.

As we can see form the above analysis, we have $\operatorname{Adv}_{B G S+}^{C D H}(W) \geq \frac{1}{n l q_{H_{2}}} \mid \operatorname{Pr}\left[\operatorname{Succ}_{4}\right]-$ $\operatorname{Pr}\left[\operatorname{Succ}_{3}\right] \mid$, that is, $\mid \operatorname{Pr}\left[\right.$ Succ $\left._{4}\right]-\operatorname{Pr}\left[\operatorname{Succ}_{3}\right] \mid \leq n l q_{H_{2}} \cdot \operatorname{Adv} v_{B G S+}^{C D H}(W)$.

Game G5. This game is as the same as $G 4$ except that in the test session the game halts if $M_{A K E}$ asks Hash query $(H)$ with the corresponding input $\left(\operatorname{sid}\left\|k_{1}\right\| \cdots \| k_{n}\right)$. Since there is no useful information about $k_{n}$ in Round 2 , the only way for the adversary to getting $k_{n}$ is to guess it with the probability $\frac{1}{2^{k}}$ and to check it using $H_{2}\left(k_{n}\right)$. Therefore,

$$
\mid \operatorname{Pr}\left[\text { Succ }_{5}\right]-\operatorname{Pr}\left[\text { Succ }_{4}\right] \mid \leq \frac{q_{H_{2}}^{2}}{2^{k}} .
$$

On the other hand, if the adversary doesn't make any Hash query for $H_{2}$ with the correct input, it will not have any advantage in distinguishing the real session key from a random one and so $\operatorname{Pr}\left[\right.$ Succ $\left._{5}\right]=\frac{1}{2}$.

Together with (2)-(7), Theorem 1 is proven.

Theorem 2 (MA security). Let $H_{1}, H_{2}$ and $H$ be three random oracles and the signature scheme used in GBS+ is UF-CMA secure, $M_{M A}$ denotes an adversary that has the ability to perform the oracle queries mentioned in Section 3. Then BGS+ is MA secure. Furthermore, the MA advantage of $M_{M A}$ $A d v_{B G S+}^{M A}\left(M_{M A}\right)$ is upper bounded by

$A d v_{B G S+}^{M A}\left(M_{M A}\right) \leq\left|n^{2} \cdot A d v_{B G S+}^{S i g}+\frac{q_{s}^{2}}{2^{k-1}}+\frac{q_{H_{1}}^{2}}{q}+\frac{q_{H_{2}}^{2}}{p}+\frac{q_{H}^{2}}{2^{\lambda}}-1\right|$, where $n$ is the number of parties; $A d v_{B G S+}^{S i g}$ denotes the advantage of a polynomial adversary against the UF-CMA security of the signature; $q_{H_{1}}, q_{H_{2}}$ and $q_{H}$ are the maximum number of times of hash queries $H_{1}, H_{2}$ and $H ; q_{s}$ represents the maximum number of queries to the Send oracles asked by the adversary.

Proof. We follow the hypothesis of the proof of Theorem 1 and define $S_{u c c_{i}}$ $(i=0,1,2,3)$ as the event that the adversary $M_{M A}$ wins the game $G i$.

Game $G 0$. Like $G 0$ in Theorem 1, this game represents the real execution. In the game, all the instances modeled as the real execution in the random oracle model. By the definition, we have

$$
A d v_{B G S+}^{M A}\left(M_{M A}\right)=\left|2 \cdot \operatorname{Pr}\left[\operatorname{Succ}_{0}\right]-1\right| .
$$

Game $G 1$. In this game, we simulate all oracles in $G 0$, except that we halt all executions in which the event Forge defined in Theorem 1 occurs. Then we have

$$
\operatorname{Pr}\left[\text { Succ }_{1}\right]-\operatorname{Pr}\left[\text { Succ }_{0}\right] \leq \operatorname{Pr}[\text { Forge }] \leq n^{2} \cdot A d v_{B G S+}^{S i g} .
$$


Game $G 2$. In this game, we simulate all oracles in $G 1$, except that we halt all executions in which the event Collision defined in Theorem 1 occurs. Consequently,

$$
\mid \operatorname{Pr}\left[\text { Succ }_{2}\right]-\operatorname{Pr}\left[\text { Succ }_{1}\right] \mid \leq \operatorname{Pr}[\text { Collision }] \leq \frac{q_{H_{1}}^{2}}{2 q}+\frac{q_{H_{2}}^{2}}{2 p}+\frac{q_{H}^{2}}{2^{\lambda+1}} .
$$

Game G3. This game is as the same as the previous game except that the simulation fails if the event Repeat defined in Theorem 1 occurs. Then we have

$$
\mid \operatorname{Pr}\left[\text { Succ }_{3}\right]-\operatorname{Pr}\left[\text { Succ }_{2}\right] \mid \leq \operatorname{Pr}[\text { Repeat }] \leq \frac{q_{s}^{2}}{2^{k}} .
$$

If $G 3$ doesn't abort, all the honest parties compute the same key. Hence $\operatorname{Pr}\left[\mathrm{Succ}_{3}\right]=0$.

Together with (8)-(11), Theorem 2 is proven.

Theorem 3 (Contributiveness). Let $H_{1}, H_{2}$ and $H$ be three random oracles and the signature scheme used in GBS+ is UF-CMA secure, $M_{C o n}$ denotes an adversary that has the ability to perform the oracle queries mentioned in Section 3 with the advantage $A d v_{B G S+}^{C o n}\left(M_{C o n}\right)$. Then BGS+ is contributive and $A d v_{B G S+}^{C o n}\left(M_{C o n}\right) \leq\left|\frac{q_{s}^{2}}{2^{k-1}}+\frac{q_{H_{2}}^{2}}{p}+\frac{q_{H}^{2}}{2^{\lambda}}-1\right|$, where $q_{H_{2}}$ and $q_{H}$ are the maximum number of times of hash queries $H_{2}$ and $H$; $q_{s}$ represents the maximum number of queries to the Send oracles asked by the adversary.

Proof. Following the proofs on Theorem 1 and Theorem 2, we construct four games from $G 0$ to $G 3$.

Game $G 0$. This game represents the real execution in the random oracle model. By the definition, we have

$$
A d v_{B G S+}^{C o n}\left(M_{C o n}\right)=\mid 2 \cdot \operatorname{Pr}\left[\text { Succ }_{0}\right]-1 \mid .
$$

Game $G 1$. In this game, we simulate all oracles as in $G 0$, except that we halt all executions in which the event Repeat defined in Theorem 1 occurs. Then we have

$$
\mid \operatorname{Pr}\left[\text { Succ }_{1}\right]-\operatorname{Pr}\left[\text { Succ }_{0}\right] \mid \leq \operatorname{Pr}[\text { Repeat }] \leq \frac{q_{s}^{2}}{2^{k}}
$$

Game $G 2$. In this game, we simulate all oracles in $G 1$, except that we halt all executions in which the event Collision 1 for input $k_{n}$ occurs. Consequently,

$$
\mid \operatorname{Pr}\left[\text { Succ }_{2}\right]-\operatorname{Pr}\left[\text { Succ }_{1}\right] \mid \leq \operatorname{Pr}[\text { Collision } 1] \leq \frac{q_{H_{2}}^{2}}{2 p} .
$$

Game G3. In this game, we simulate all oracles in $G 2$, except that we halt all executions in which the event Collision2 for input $\left(\operatorname{sid}\left\|k_{1}\right\| \cdots \| k_{n}\right)$ occurs Consequently,

$$
\mid \operatorname{Pr}\left[\text { Succ }_{3}\right]-\operatorname{Pr}\left[\text { Succ }_{2}\right] \mid \leq \operatorname{Pr}[\text { Collision2 }] \leq \frac{q_{H}^{2}}{2^{\lambda+1}} .
$$

If $G 3$ doesn't abort, the output of the random oracle $H$ is uniformly distributed. Hence, $\operatorname{Pr}\left[\mathrm{Succ}_{3}\right]=0$.

Together with (12)-(15), Theorem 3 is proven. 


\section{Conclusion}

We analyze GBG model and find that this model doesn't consider the ephemeral key leakage resistance property. Considering this drawback, we extend GBG model to the following extent: the only corruption powers we don't give an adversary in the game are that it is not allowed to reveal both the long-term key and the ephemeral key of a legitimate party simultaneously. We then introduce a new GKA protocol called BGS+ protocol based on BGS protocol which is provably secure in GBG model but cannot resist ephemeral key leakage attack and prove that it is secure in the new model. As far as we know, this is the first provably secure GKA protocol in the strongest security model.

As for limitations, we didn't take the efficiency into account. A natural direction for further research is the design of provably secure protocols with lower computational cost and communication cost. Additionally, the design of GKA protocols in standard models where the security of the protocols isn't dependent on the security of random oracles is another imperative future research consideration.

\section{Acknowledgement}

This work is partially supported by the Specialized Research Fund for the Doctoral Program of Higher Education (No. 200802480019) and the National Natural Science Foundation of China (No.61073150).

\section{References}

1. Manulis, M.: Provably Secure Group Key Exchange. Volume 5 of IT Security. Europäischer Universitätsverlag, Berlin, Bochum, Dülmen, London, Paris (August 2007) Available at http://www.manulis.eu/phd.html. Last accessed on 29 May 2008.

2. Bellare, M., Rogaway, P.: Entity Authentication and Key Distribution. In: Advances in Cryptology-CRYPTO'93. Volume 773 of LNCS., Springer (1993) 232-249

3. Canetti, R., Krawczyk, H.: Analysis of Key-Exchange Protocols and Their Use for Building Secure Channels. In: Advances in Cryptology - EUROCRYPT'01. Volume 2045 of LNCS., Springer (2001) 453-474

4. LaMacchia, B.A., Lauter, K., Mityagin, A.: Stronger Security of Authenticated Key Exchange. In Susilo, W., Liu, J.K., Mu, Y., eds.: Provable Security, First International Conference, ProvSec 2007. Volume 4784 of LNCS., Springer (2007) $1-16$

5. Abdalla, M., Fouque, P.A., Pointcheval, D.: Password-Based Authenticated Key Exchange in the Three-Party Setting. In Vaudenay, S., ed.: Public Key Cryptography-PKC'05. Volume 3386 of LNCS., Springer (2005) 65-84

6. Wang, W., Hu, L.: Efficient and Provably Secure Generic Construction of ThreeParty Password-Based Authenticated Key Exchange Protocols. In Barua, R., Lange, T., eds.: Progress in Cryptology-INDOCRYPT'06. Volume 4329 of LNCS., Springer (2006) 118-132 
7. Yoneyama, K.: Efficient and Strongly Secure Password-Based Server Aided Key Exchange (Extended Abstract). In Chowdhury, D.R., Rijmen, V., Das, A., eds.: Progress in Cryptology-INDOCRYPT'08. Volume 5365 of LNCS., Springer (2008) $172-184$

8. Blake-Wilson, S., Johnson, D., Menezes, A.: Key Agreement Protocols and Their Security Analysis. In: Cryptography and Coding, 6th IMA International Conference. Volume 1355 of LNCS., Springer (1997) 30-45

9. Bresson, E., Chevassut, O., Pointcheval, D., Quisquater, J.J.: Provably authenticated group Diffie-Hellman key exchange. In: CCS'01: Proceedings of the 8th ACM conference on Computer and Communications Security, ACM (2001) 255-264

10. Bresson, E., Chevassut, O., Pointcheval, D.: Provably Authenticated Group DiffieHellman Key Exchange - The Dynamic Case. In: Advances in CryptologyASIACRYPT'01. Volume 2248 of LNCS., Springer (2001) 290-309

11. Bresson, E., Chevassut, O., Pointcheval, D.: Dynamic Group Diffie-Hellman Key Exchange under Standard Assumptions. In: Advances in CryptologyEUROCRYPT'02. Volume 2332 of LNCS., Springer (2002) 321-336

12. Katz, J., Shin, J.S.: Modeling insider attacks on group key-exchange protocols. In: Proceedings of the 12th ACM Conference on Computer and Communications Security-CCS'05, ACM (2005) 180-189

13. Bohli, J.M., Gonzalez Vasco, M.I., Steinwandt, R.: Secure group key establishment revisited. Int. J. Inf. Sec. 6(4) (2007) 243-254

14. Bresson, E., Manulis, M.: Securing Group Key Exchange against Strong Corruptions. In: Proceedings of ACM Symposium on Information, Computer and Communications Security (ASIACCS'08), ACM Press (2008) 249-260

15. Gorantla, M.C., Boyd, C., González Nieto, J.M.: Modeling Key Compromise Impersonation Attacks on Group Key Exchange Protocols. In Jarecki, S., Tsudik, G., eds.: Public Key Cryptography-PKC'09. Volume 5443 of LNCS., Springer (2009) $105-123$

16. Boyd, C., Nieto, J.M.G.: Round-Optimal Contributory Conference Key Agreement. In: Public Key Cryptography-PKC'03. Volume 2567 of LNCS., Springer (2003) 161-174

17. Al-Riyami, S.S., Paterson, K.G.: Tripartite Authenticated Key Agreement Protocols from Pairings. In: Cryptography and Coding, 9th IMA International Conference. Volume 2898 of LNCS., Springer (2003) 332-359

18. Bresson, E., Chevassut, O., Essiari, A., Pointcheval, D.: Mutual Authentication and Group Key Agreement for Low-Power Mobile Devices. In: Proc. of MWCN'03, World Scientific Publishing (October 2003) 59-62

19. Manulis, M., Suzuki, K., Ustaoglu, B.: Modeling Leakage of Ephemeral Secrets in Tripartite/Group Key Exchange. In Lee, D., Hong, S., eds.: Information, Security and Cryptology-ICISC'09, Revised Selected Papers. Volume 5984 of LNCS., Springer (2010) 16-33

20. Shoup, V.: Sequences of games: a tool for taming complexity in security proofs. Cryptology ePrint Archive, Report 2004/332 (2004) http://eprint.iacr.org/. 